\title{
Dynamic evaluation of lung involvement during coronavirus disease-2019 (COVID-19) with quantitative lung CT
}

\author{
Chun $\mathrm{Ma}^{1}{ }^{1}$ (D) Xiao-Ling Wang ${ }^{1} \cdot$ Dong-Mei Xie $^{1} \cdot$ Yu-Dan $\mathrm{Li}^{1} \cdot$ Yong-Ji Zheng $^{1} \cdot$ Hai-Bing Zhang ${ }^{1} \cdot$ Bing Ming $^{1}$
}

Received: 28 June 2020 / Accepted: 24 September 2020 / Published online: 10 October 2020

(C) American Society of Emergency Radiology 2020

\begin{abstract}
Purpose To identify and quantify lung changes associated with coronavirus disease-2019 (COVID-19) with quantitative lung CT during the disease.

Methods This retrospective study reviewed COVID-19 patients who underwent multiple chest CT scans during their disease course. Quantitative lung CT was used to determine the nature and volume of lung involvement. A semi-quantitative scoring system was also used to evaluate lung lesions.

Results This study included eighteen cases ( 4 cases in mild type, 10 cases in moderate type, 4 cases in severe type, and without critical type cases) with confirmed COVID-19. Patients had a mean hospitalized period of $24.1 \pm 7.1$ days (range: 14-38 days) and underwent an average $\mathrm{CT}$ scans of $3.9 \pm 1.6$ (range: $2-8$ ). The total volumes of lung abnormalities reached a peak of $8.8 \pm 4.1$ days (range: $2-14$ days). The ground-glass opacity (GGO) volume percentage was higher than the consolidative opacity (CO) volume percentage on the first $C$ T examination $(Z=2.229, P=0.026)$, and there was no significant difference between the GGO volume percentage and that of $\mathrm{CO}$ at the peak stage $(Z=-0.628, P=0.53)$. The volume percentage of lung involvement identified by AI demonstrated a strong correlation with the total CT scores at each stage $(r=0.873, P=0.0001)$.

Conclusions Quantitative lung CT can automatically identify the nature of lung involvement and quantify the dynamic changes of lung lesions on CT during COVID-19. For patients who recovered from COVID-19, GGO was the predominant imaging feature on the initial CT scan, while GGO and $\mathrm{CO}$ were the main appearances at peak stage.
\end{abstract}

Keywords Coronavirus $\cdot$ Artificial intelligence $\cdot$ Tomography $\cdot$ X-ray $\cdot$ Lung $\cdot$ Pneumonia $\cdot$ viral

\section{Introduction}

A new coronavirus was first detected in Wuhan, Hubei Province, China, in early December 2019, and then termed severe acute respiratory syndrome coronavirus 2 (SARS-CoV-2) [1]. The disease has affected the whole world, and it has been named coronavirus disease-2019 (COVID-19). The WHO declared the COVID-19 constituted a public health emergency of international concern on January 30, 2020. As of June 28,

Electronic supplementary material The online version of this article (https://doi.org/10.1007/s10140-020-01856-4) contains supplementary material, which is available to authorized users.

Bing Ming

mingbing.mb@163.com

1 Department of Radiology, People's Hospital of Deyang City, Taishanbei 173, Jinyang District, Deyang 618000, China
2020, confirmed cases with COVID-19 worldwide had surpassed 9,000,000, while the death toll has topped 495,000. Similar to other coronavirus pneumonia, COVID-19 mainly causes alveolar edema with hemorrhage, bronchiolitis, alveolitis, and pulmonary interstitial fibrosis [2]. With the gradual understanding of the disease, CT is reserved for hospitalized, symptomatic patients for assessing the progression course of COVID-19 [3-6]. Recent studies have used semi-quantitative methods to observe the lung changes during COVID19 from the onset of symptoms to recovery with CT images [7, 8]. However, such a semi-quantitative method is time-consuming and has a significant subjective bias. Artificial intelligence (AI) has been used in many clinical fields in recent years, especially for the quantitative evaluation of lung diseases [9-11]. This study aimed to assess the changes associated with COVID19 on chest CT through the course of the disease until the discharge of patients with AI. 


\section{Material and methods}

The Ethics of Committees of People's Hospital of Deyang City approved this retrospective study, and informed consent for this retrospective study was waived.

\section{Patients selection}

Patients with confirmed COVID-19 were reviewed coming from Deyang City from January 26, 2020, to June 15, 2020. The diagnostic criteria were based on the preliminary diagnosis and treatment protocol issued by the National health commission of the People's Republic of China:

1. Suspected cases were considered if having any one of the epidemiological histories or meeting any two of the clinical manifestations: (1) travel history or residence history in Wuhan area or other areas with continuous local case transmission within 14 days before the onset of the symptoms; (2) history of having contact with other patients with fever or respiratory symptoms coming from Wuhan or other areas where local cases continued to spread within 14 days before the onset of illness; (3) aggregative onset or epidemiological association with COVID-19 patients; (4) fever; (5) having the imaging characteristics of COVID-19; (6) the total number of white blood cells is normal or decreased, or the lymphocyte count is decreased in the early stage of onset.

2. Cases confirmed with real-time fluorescent polymerase chain reaction (RT-PCR) of respiratory specimens or blood specimens proved detection of the novel coronavirus; virus gene sequencing of respiratory or blood specimen was highly homologous to the new coronavirus (11). Patients met the following conditions could be discharged: (1) body temperature returned to normal for more than 3 days; (2) significant improvement in respiratory symptoms; (3) lung imaging showed a marked improvement in acute exudative lesions of the lungs; (4) two consecutive sputum, nasopharyngeal swabs, or other respiratory specimens were negative for nucleic acid testing, and the sampling interval was more than $24 \mathrm{~h} \mathrm{[4].}$

All consecutive 18 patients from $* * * *$ City were admitted to the People's Hospital of **** City, the regional medical center of $* * * *$ City, and isolated in the separate infectious disease hospital area for treatment. No patients were excluded.

\section{Chest CT protocol}

Non-contrast $\mathrm{CT}$ scans were performed using a single-source CT scanner (Emotion 16, VB41A, Siemens Healthcare, Forchheim, Germany), which was dedicated to a separated COVID-19 ward. The patients were scanned with a single inspiratory phase in the supine position. A specially tailored low-dose protocol was employed for COVID-19 cases for reducing the amount of patient exposure to ionizing radiation. The tube voltage was $110 \mathrm{kVp}$, while reference mAs was 70 $\mathrm{mAs}$ with automatic tube current modulation. A matrix size of $512 \times 512(1.5-\mathrm{mm}$ slice thickness and $1.5-\mathrm{mm}$ increment $)$ was performed to reconstruct $\mathrm{CT}$ images. B90f kernel and B31f kernel were used for lung kernel and mediastinal kernel, respectively. The iterative reconstruction was not available in this version of CT system. Window level of - 600600 Hounsfield unit (HU) and window width of $1600 \mathrm{HU}$ were set for lung window setting, while window level of $40 \mathrm{HU}$ and window width of $350 \mathrm{HU}$ for mediastinum window setting. The mean dose-length product was $115.6 \pm 22.9 \mathrm{mGy}^{2} \mathrm{~cm}$.

\section{CT images review}

Anonymous data were transmitted to the Dr. Wise system (version v1.3.0.1, Beijing Deepwise \& League of PhD Technology Co. Ltd, China; the system is currently under the China Food and Drug Administration review process.) for automatic detection and segmentation. The Dr. Wise system performed lung segmentation, lesion segmentation and detection, and volume calculation using a dedicated multitask deep learning algorithm developed for pulmonary pneumonia based on both regular-dose protocol and low-dose protocol. All those algorithms are based on supervised training of deep neural networks, i.e., based on training neural networks on large-scale annotated data (over ten thousand cases), which include a wide range of varieties in the sense of manufactures, acquisition parameters, reconstruction kernel, and slice thickness. Thus, the trained model is robust to all these changes. A variant of the convolutional MVP-Net [12] is exploited to achieve automatic detection of the pneumonia-related symptom regions, like GGO and $\mathrm{CO}$. The categorization of GGO and $\mathrm{CO}$ is not based on thresholding Hounsfield units. It is based on supervised training with deep neural networks. Experienced radiologists firstly label GGO and CO areas, and then the deep neural networks are trained with acquired labels. Since radiologists tend to inspect multiple windows to obtain an accurate diagnosis, the MVP-Net takes advantage of such domain knowledge and employs a multi-view feature pyramid network to extract features from images rendered with varying window widths and window levels. Afterward, 3D U-Net [13] with pseudo-3D convolution was introduced to segment voxels that represented the abnormality in the detected regions. Thus, we could acquire the delineation of the pneumonia-related symptom regions.

For lung lesions identified on Dr. Wise, the reviewers recorded volumes of ground-glass opacity (GGO), consolidative opacity (CO), and the total abnormalities volume, respectively. The abnormality volumes as a percentage of total lung volume also were recorded for a series of CT 
examinations from admission to discharge. Comparisons of the percentage of the volume of GGO and $\mathrm{CO}$ on the first CT scan, the last CT scan, and the CT scan at the peak stage of the disease course were performed.

The CT images were transmitted to the institutional digital database system (Infinitt, Shanghai, China). Two cardiothoracic radiologists (C.M with 15 years of experience and HBZ) subjectively evaluated images on a 30 -in. $8 \mathrm{M}$ color LCD. If the two radiologists failed to reach a consensus, a third cardiothoracic radiologist (B.M) with 20 years of experience determined the final decision. Based on the abnormalities of lobes related to COVID-19 (e.g., GGO, CO, reticulation, nodules, interlobular septal thickening, and fibrosis), reviewers assessed lung involvement in lung window images using a semi-quantitative scoring system [6]. Visually scoring each of the five lobes was performed as 0 for none; 1 for $1-25 \%$ involvement; 2 for $26-49 \%$ involvement; 3 for $50-75 \%$ involvement; and 4 for $76-100 \%$ involvement. The scores for each lobe were summed to reach a total severity score ranging from 0 to 20. All follow-up CT images were evaluated for all patients to observe the lung change over time until recovery. The spent time was recorded from the start of scoring to completion for every case.

\section{Statistical analysis}

Depending on the normality of distribution, the Student $t$ test, ANOVA, Wilcoxon test, or Mann-Whitney $U$ test was performed for continuous variables assessed by the ShapiroWilk test. Continuous data were presented as mean \pm standard deviation (minimum-maximum) or median (minimum-maximum). Pearson correlation was used to evaluate correlations between scores and the abnormalities volume percentage. SPSS 22.0 (version 22.0, IBM, Armonk, NY, USA) and GraphPad Prism 7 (GraphPad Software Company, San Diego, CA, USA) were used in statistical analyses. A two-sided $P$ value of less than 0.05 was considered a significant difference.

\section{Results}

\section{Patient characteristics}

A total of 18 patients were included in this study ( 9 men and 9 women) with an average age of $45.7 \pm 20.1$ years ( 46 days to 76 years) (Table 1 ). The most common initial symptoms were cough $(50 \%)$ and fever $(72.2 \%)$. Four patients $(4 / 18,22 \%)$ were classified into mild type without any abnormalities on chest CT scans. Ten patients $(10 / 18,55.6 \%)$ were in moderate type and four patients $(4 / 18,22 \%)$ in severe type according to the preliminary diagnosis and treatment protocol issued by the National Health Commission of the People's Republic of
China [13]. There were no cases of critical type in $* * * *$ City (a city located in the southwest of China with 3.9 million population). The most frequent laboratory investigations were mildly elevated hs-CRP (hypersensitive C-reactive protein) $(14.9 \pm 18 \mathrm{mg} / \mathrm{L})$ and mildly decreased lymphocyte percentage in moderate types $(17.4 \%)$ and severe types $(15.5 \%)$ (Table 1). The average interval from the onset of initial symptoms to the first CT scan was $4.4 \pm 5.1$ days (range: $1-18$ days). The average hospitalization days were $24.1 \pm 7.1$ days (range: 14-38 days). The number of CT scans per patient was $3.9 \pm 1.6(2-8)$, and the total number of CT scans was 70 .

\section{Lung abnormality evaluation}

\section{Al evaluation}

The Dr. Wise system automatically identified lung abnormalities related to COVID-19 on lung area (Fig. 1). This system classified lung abnormalities into GGO or consolidation. It measured the volume of these lesions, furthermore, developed a curve to show the trend of changes in lung lesions with data of the series follow-up CT scans. The total volumes of lung involvement reached a peak of $8.8 \pm 4.1$ days (range: $2-14$ days). The volumes of lung abnormalities at the peak stage from patients of severe type were higher than those of patients from moderate type $(Z=-2.828, P=0.002)$ (Fig. 2). Two cases in severe type developed the second lung lesions volume peak around 22 days after the onset of symptoms, along with symptoms deteriorated. The GGO volume percentage (median $=1.4 \%$, range: $0.01-26.5 \%)$ was higher than the $\mathrm{CO}$ volume percentage (median $=0.49 \%$, range: 0 $4.5 \%)$ on the first CT examination $(Z=2.229, P=0.026)$ (Fig. 3a). No significant difference appeared between the GGO volume percentage $($ median $=2.4 \%$, range: $032.6 \%$ ) and the $\mathrm{CO}$ volume percentage (median $=3.4 \%$, range: $0.1-45.5 \%)$ at the peak stage $(Z=-0.628, P=0.53)$ (Fig. $3 b)$. There was no significant difference between the GGO volume percentage (median $=0.06 \%$, range: 0 $4.4 \%$ ) and the $\mathrm{CO}$ volume percentage (median $=0.9 \%$, range: $0-24.1 \%$ ) at the last $\mathrm{CT}$ examination before discharge $(Z=-1.821, P=0.069)$ (Fig. 3c). The total volume percentage of abnormalities on the $\mathrm{CT}$ images at the peak stage (median $=5.2 \%$, range: $1.7-47.9 \%$ ) was higher than that on the initial $\mathrm{CT}$ scans (median $=1.9 \%$, range: $0.2-26.8 \%)(Z=-3.621, P<0.001)$ and that on the last CT scans $($ median $=1.8 \%$, range: $0.03-28.5 \%)(Z=-$ 3.154, $P<0.002$ ) (Fig. 3d).

\section{Semi-quantitative scoring system and Al}

The total CT scores peaked at $7.8 \pm 4.3$ days after the first symptom appeared. This peak time of the total CT scores 
Table 1 Baseline characteristics of the patient cohort

\begin{tabular}{|c|c|c|c|c|}
\hline & Total $(n=18)$ & Mild type $(n=4)$ & Moderate type $(n=10)$ & Severe type $(n=4)$ \\
\hline \multicolumn{5}{|l|}{ Patient characteristics } \\
\hline Returning from Wuhan & $9(50 \%)$ & $0(0)$ & $5(50 \%)$ & $4(100 \%)$ \\
\hline Age (years) & $45.7 \pm 20.1(0.1-76)^{*}$ & $21(0.1-45)^{\#}$ & $46(30-76){ }^{\#}$ & $51(40-71)^{\#}$ \\
\hline Male & $9(50 \%)$ & $2(50 \%)$ & $5(50 \%)$ & $2(50 \%)$ \\
\hline \multicolumn{5}{|l|}{ Initial symptoms } \\
\hline Headache & $2(11.1 \%)$ & $0(0)$ & $1(10 \%)$ & $1(25 \%)$ \\
\hline Cough & $9(50 \%)$ & $1(25 \%)$ & $6(60 \%)$ & $2(50 \%)$ \\
\hline Fever & $13(72.2 \%)$ & $2(50 \%)$ & $7(70 \%)$ & $4(100 \%)$ \\
\hline Fatigue & $2(11.1 \%)$ & $0(0)$ & $1(10 \%)$ & $1(25 \%)$ \\
\hline Diarrhea & $5(27.8 \%)$ & $2(50 \%)$ & $3(30 \%)$ & $0(0)$ \\
\hline \multicolumn{5}{|l|}{ Laboratory investigations } \\
\hline White blood cell count (G/L) & $4.9 \pm 2.1(1.3-9.9)^{*}$ & $4.1(3.7-9.9)^{\#}$ & $5.2(3.3-7.1)^{\#}$ & $3.3(1.3-4.1)^{\#}$ \\
\hline Neutrophil count $(\mathrm{G} / \mathrm{L})$ & $3.5 \pm 1.8(0.7-7.4)^{*}$ & $2.3(0.9-7.4)^{\#}$ & $4.0(1.7-5.3)^{\#}$ & $2.2(0.7-3.4)^{\#}$ \\
\hline Lymphocyte count (G/L) & $1.0 \pm 0.4(0.5-1.8)^{*}$ & $1.7(0.7-6.3)^{\#}$ & $1.1(0.5-1.5)^{\#}$ & $0.7(0.5-1.2)^{\#}$ \\
\hline Lymphocyte percentage (\%) & $22.5 \pm 9.2(12-39.3)^{*}$ & $43.5(19.9-58.8)$ & $17.4(14.1-39.3)^{\#}$ & $15.5(12-25)^{\#}$ \\
\hline Monocyte percentage (\%) & $8.9 \pm 3.3(4.9-17.3)^{*}$ & $6.8(2.9-10.8)^{\#}$ & $5.3(3.3-7.1)^{\#}$ & $3.3(1.3-4.1)^{\#}$ \\
\hline hs-C-reactive protein (mg/L) & $14.9 \pm 18(0.4-57.5)^{*}$ & $2.1(1-8.6)^{\#}$ & $4.8(0.4-24.3)^{\#}$ & $37.2(17.6-57.5)^{\#}$ \\
\hline Myoglobin $(\mu \mathrm{g} / \mathrm{L})$ & $36.8 \pm 14.7(14.7-67.1)^{*}$ & $35.8(19.4-)-55.8)^{\#}$ & $35.4(14.7-67.1)^{\#}$ & $39(27.9-39.1){ }^{\#}$ \\
\hline Troponin $(\mu \mathrm{g} / \mathrm{L})$ & $0.05 \pm 0.03(0-0.2)^{*}$ & $0.005(0-0.19){ }^{\#}$ & $0(0-0.02)^{\#}$ & $0.14(0-37.7)^{\#}$ \\
\hline Oxygenation index $(\mathrm{mm} \mathrm{Hg})^{\delta}$ & N/A & N/A & $\mathrm{N} / \mathrm{A}$ & $190.5(111-400)^{\#}$ \\
\hline Onset of initial symptoms to the first CT scan (days) & $4.4 \pm 5.1(0-18) *$ & $10.3 \pm 6.8(4-18)^{*}$ & $2.8 \pm 2.7(1-9)^{*}$ & $1.5 \pm 1.6(0-4)^{*}$ \\
\hline Numbers of CT scans & $3.9 \pm 1.6(2-8) *$ & $2.3 \pm 0.5(2-3)^{*}$ & $3.7 \pm 0.8(3-5)^{*}$ & $6 \pm 1.8(4-8)^{*}$ \\
\hline The interval between adjacent scans (days) & $5.6 \pm 2.7(1-17) *$ & $10 \pm 4.8(7-17)^{*}$ & $5.7 \pm 2.7(1-11)^{*}$ & $4.6 \pm 0.9(2-7)^{*}$ \\
\hline The hospitalized period (days) & $24.1 \pm 7.1(14-38)^{*}$ & $21 \pm 7.1(14-30)^{*}$ & $23.4 \pm 6.3(17-36)^{*}$ & $28.5 \pm 8.6(19-38)^{*}$ \\
\hline
\end{tabular}

Note: Quantitative data were presented as mean \pm standard deviation (minimum-maximum)* or median (minimum-maximum) ${ }^{\#}$, while the counting data were presented as count (percentage of the total). Oxygenation index was only obtained from severe patients ${ }^{\delta}$. Oxygenation index was defined by the arterial partial pressure of oxygen $\left(\mathrm{PaO}_{2}\right)$ /oxygen fraction ratio of inhalation gas $\left(\mathrm{FiO}_{2}\right)$

$N / A$, not available

was close to that of the volumes of lung abnormalities $(Z=-$ $1.045, P=0.306)$. The percentage of lung involvement volume identified by AI demonstrated a strong correlation with the total CT scores at each stage $(r=0.873, P=0.0001)$ (Fig. $4)$. The lung involvement volume had a significant correlation with the total CT scores $(r=0.807, P=0.0001)$ (Supplementary Fig. 1), while a strong negative correlation with the oxygenation index $(r=0.863, P=0.0001)$ (Supplementary Fig. 2) in the four severe cases. Laboratory tests, including white blood cell count, percentage of lymphocyte, percentage of neutrophils, and hs-CRP, showed no significant correlation with the percentage of lung abnormalities volume (Supplementary Fig. 3). The mean time spent was $40.6 \pm 16.8 \mathrm{~s}$ on scoring lung lesions for all CT examinations. The results of identified lung abnormalities were instantly available when reviewers turned on the operation interface of Dr. Wise because the data had been preloaded and processed.

\section{Discussion}

This study was to identify the lung involvement on chest CT with AI and to observe the dynamic change during the disease course associated with COVID-19. All 18 cases with COVID19 coming from $* * * *$ City had been discharged with hospitalization days of $24.1 \pm 7.1$ days. The Dr. Wise system could determine the nature of lung abnormalities associated with COVID-19 to be GGO or CO and yield the volume of the lesions almost instantly. The quantification of lung involvement and the rapid and accurate results are conducive to the efficient and precise assessment of the patient's situation in a pandemic situation.

The Dr. Wise system we applied in this study strengthened the detection of typical signs in the new coronavirus pneumonia. Based on the consolidation shadow and ground-glass density shadow outlined by the doctor, these two abnormal signs were automatically detected and segmented with the U-Net 


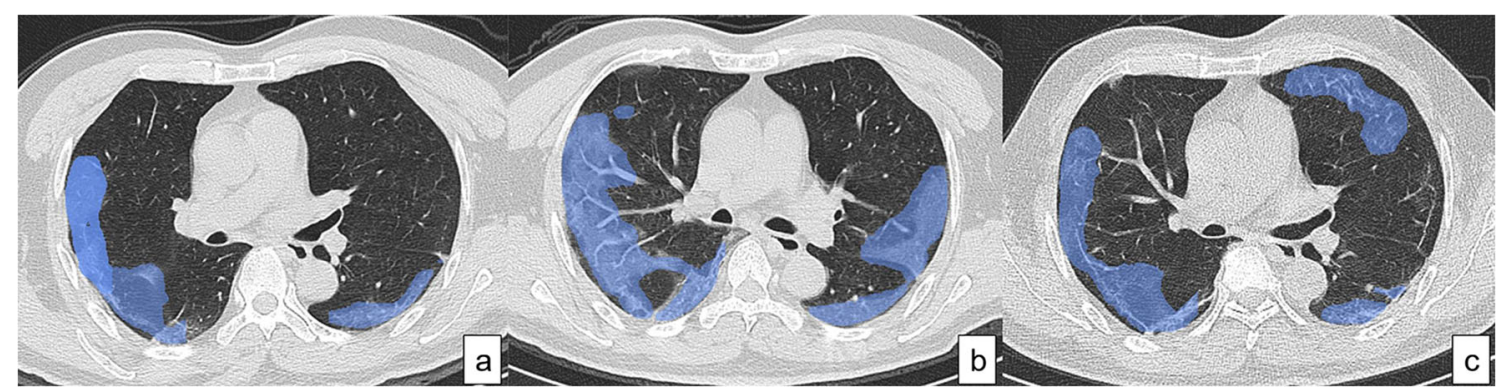

Fig. 1 A 54-year old male with COVID-19. a AI automatically identifies GGO on the initial CT scan on Dr. Wise system; b The volume of GGO increases on $\mathrm{CT}$ scan at the peak stage; c The volume of abnormalities

model using the 3D context modeling [14]. Specifically, the model took continuous multiple layers in the $\mathrm{CT}$ images as the model input, and the outline of typical abnormal signs is obtained through model prediction. At the same time, the same neural network could also be used to accurately segment the lung lobe and the lung segment area, thereby calculating the specific volume of the lesion and its volume proportion in the lung lobe.

In our cohort, mild type patients (4 cases) confirmed by RT-PCR had no lung involvement on chest CT with or without mild symptoms. These four cases who underwent at least 2 $\mathrm{CT}$ scans showed no abnormalities on chest $\mathrm{CT}$ images. The remaining 14 patients with lung involvement underwent $\mathrm{CT}$ scans at least 3 times. The series of imaging examinations provided details of the change of features for lung involvement induced by COVID-19. On the first CT examination, all moderate and severe types of cases had lung involvement. The volume percentage of GGO was higher than that of CO. In line with published literature [7, 8], GGO was the characteristic imaging feature on the initial CT scan. The SARS-CoV-2 belongs to the same coronavirus family as the severe acute respiratory syndrome coronavirus (SARS-CoV). Coronavirus S protein (spike glycoprotein) has a strong affinity with human cells' angiotensin-converting enzyme 2 (ACE2) [15]. ACE2 is mainly expressed on the surface of type II alveolar cells in the respiratory system [16]; hence, COVID19 involves mostly the lower respiratory tract and occurs relieves on the last CT scan before discharge. COVID-19, coronavirus disease 2019; GGO, ground-glass opacity

GGO in a similar fashion of severe acute respiratory syndrome at the early stage of the disease [17].

In the published literature, AI has shown the ability to quantify COVID-19 lung lesions and stratify or evaluate the patient's condition in the context of the global pandemic of COVID-19 quickly and accurately [18-20]. The volumes of lung involvement in this group reached a peak of around 8.8 days (ranging from 2 to 14 days) after onset. This development trend of COVID-19 in patients classified into moderate and severe types was similar to the research by Pan and colleagues [7]. This study also revealed that $\mathrm{CO}$ and GGO were remarkable imaging features at the peak stage on chest $\mathrm{CT}$ for COVID-19 patients [7, 21]. Most lung lesions were gradually resolved in subsequent follow-up CT scans, except for two cases in severe type rebounded around 22 days after onset of symptoms along with worsening symptoms. The last chest CT scans before discharge showed that the percentage of lung lesions was significantly smaller than that of the peak period. These dynamic image assessment data provided objective evidence for treatment efficacy evaluation.

A scoring system has been used by previous studies to assess lung involvement of COVID-19 based on chest CT $[7,8]$. This system allows the semi-quantitative evaluation of lung lesions of COVID-19 and indicates the lung involvement of the disease. Identification and measurement of lung involvement automatically with $\mathrm{AI}$ in this study demonstrated a strong correlation with the scoring system $(r=0.873, P=$
Fig. 2 Dynamic changes of the total lung involvement volume for each patient from the onset of initial symptoms to the last $\mathrm{CT}$ scan before discharge. Case A, case $\mathrm{B}$, case $\mathrm{C}$, and case $\mathrm{D}$, classified into severe type, visually show more lung involvement at the peak stage than the 10 cases in moderate type (color should be used for any figures in print)

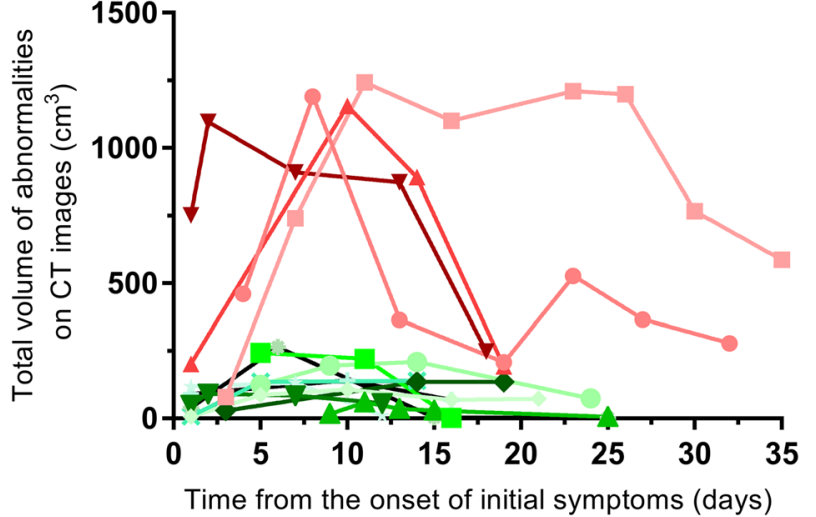

Case

No.

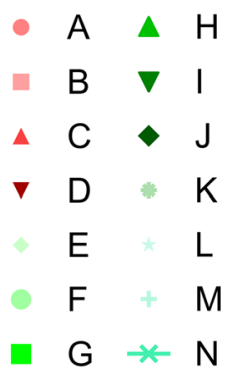


Fig. 3 Dynamic changes in the lung abnormality volume during COVID-19. a The GGO volume percentage is higher than the $\mathrm{CO}$ volume percentage on the first chest CT scan; b, c No significant difference between the GGO and $\mathrm{CO}$ volume percentage on the peak stage and the last CT scan; $d$ The total lung involvement volume percentage is higher than that of the first and last CT scans, no significant difference between the first and last $\mathrm{CT}$ scans. COVID-19, coronavirus disease 2019; GGO, ground-glass opacity; $\mathrm{CO}$, consolidative opacity
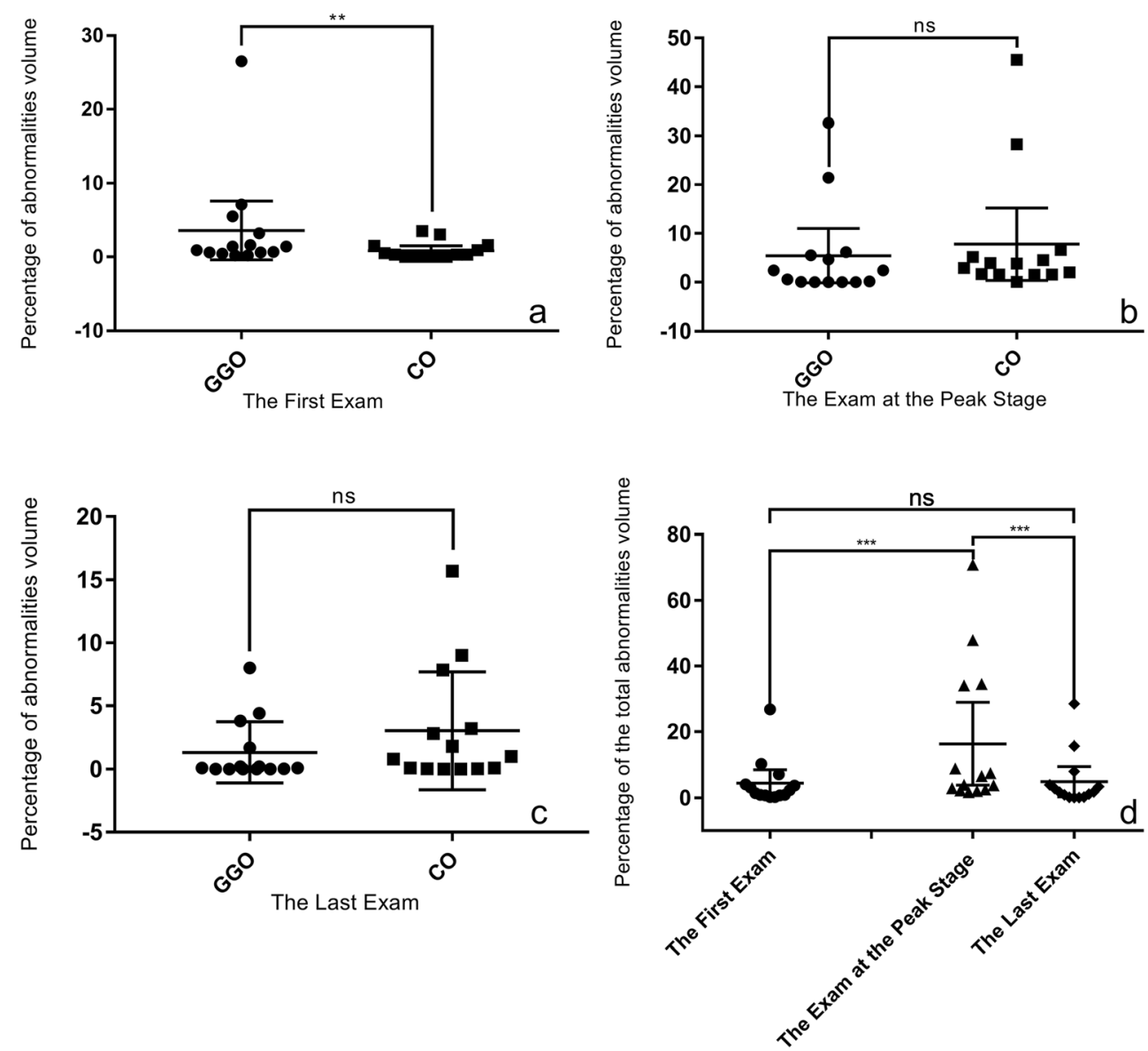

0.0001). Both evaluated the ratio of lesions volume to lung volume. Furthermore, in the four severe cases, we observed a negative correlation between the lung involvement volume and the oxygenation index. Though the oxygenation index was only assessed in severe patients, this result indicated that the severity of lung involvement on images would shed light on evaluating the condition of COVID-19 patients. Besides,

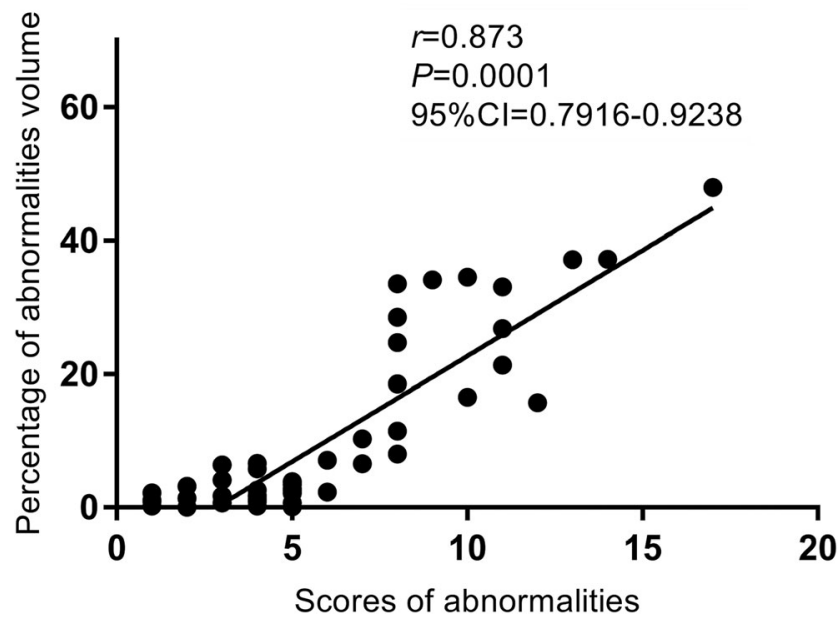

Fig. 4 The linear correlation existing between the lung abnormalities volume percentage and scores of the lung abnormalities
AI has two advantages. The first is that AI performed the quantitative evaluation of lung lesions, which is more accurate than the subjective assessment of lung involvement that the scoring system used. The second is AI evaluates COVID-19 lung abnormalities more quickly, compared with semiquantitative scores. AI can obtain measurement results almost instantaneously and can provide the dynamic trend of lung involvement for the disease based on a series of follow-up imaging data, which is far superior to the semi-quantitative scoring system. Despite the fact that the use of chest CT for diagnosing COVID-19 is currently not recommended by the American College of Radiology (ACR) [6], the National Health Commission of the People's Republic of China still has recommended that the chest $\mathrm{CT}$ should be used for disease monitoring [4]. Our study indicated that chest CT combined with AI could well evaluate the dynamic lung changes during the COVID-19 course.

This work has a few limitations. Firstly, AI only identified lung lesions of COVID-19 as GGO and CO in this study. It classified crazy-paving pattern into GGO, although this feature was less frequent on initial CT images [22]. Previous studies have shown that three main categories of CT findings are seen in association with the progression of COVID-19: GGO, crazy-paving pattern, and $\mathrm{CO}[8,23,24]$. In the early 
stage of the disease, GGO was the main finding seen in the lower lobes on CT images. As the disease progressed, the crazy-paving pattern appeared together with the diffuse GGO. Then, GGO and the crazy-paving pattern progressed to consolidation, especially in patients with a long interval from symptom onset to diagnosis and treatment [25]. Sun and colleagues [26] reported that CT quantitative analysis might be an effective and valuable method for assessing the severity of COVID-19. They classified the lung lesions into GGO and CO by using AI, which significantly correlated with pathologic patterns of the lung at early and peak stages during COVID-19. In our work, the total lesion percentage on CT quantitatively represents the percentage of lung parenchyma involvement, demonstrating evidence of abnormalities as a whole and assesses of the total extent of the disease. AI also identified fibrosis as $\mathrm{CO}$. Fibrosis usually appeared in the later stages of the disease. AI sometimes overestimated or underestimated the volume of lung lesions. Secondly, the number of COVID-19 cases in **** City was only 18 , which limited the sample size and lack of critical cases.

\section{Conclusions}

AI can automatically identify lung involvement associated with COVID-19 and quantitatively assess the dynamic changes of lung abnormalities during the disease. For patients who recovered from COVID-19, GGO was the predominant imaging feature on the initial CT scan, and the prevalent appearances at peak stage were GGO and CO. AI can actively facilitate clinical decision-making in the setting of the current COVID-19 global pandemic with an urgent need to manage a vast number of patients.

Authors' contributions All authors contributed to the study conception and design. Material preparation, data collection, and analysis were performed by Ma Chun, Xiao-Ling Wang, Dong-Mei Xie, Yu-Dan Li, HaiBing Zhang, and Yong-Ji Zheng. The first draft of the manuscript was written by Ma Chun and supervised by Bing Ming. Statistics analysis was performed by Chun Ma. All authors commented on previous versions of the manuscript. All authors read and approved the final manuscript.

Funding This work was supported by the "Research \& Development Project of New Coronavirus Infection Pneumonia Prevention Technology" in Deyang City (grant number: FY202006).

\section{Compliance with ethical standards}

Conflict of interest The authors declare that they have no conflict of interest.

Ethics approval This study was conducted retrospectively from data obtained for clinical purposes. The study received Institutional Review Board approval.
Consent to participate A waiver of informed consent was granted for this retrospective study.

Consent to publish The included CT images are not necessary to obtain consent as without any identifying information.

Code availability Not applicable.

\section{References}

1. Huang C, Wang Y, Li X, Ren L, Zhao J, Hu Y et al (2020) Clinical features of patients infected with 2019 novel coronavirus in Wuhan, China. Lancet 395(10223). https://doi.org/10.1016/S01406736(20)30183-5

2. W. Luo, H. Yu, J. Gou, X. Li, Y. Sun, J. Li, et al. Clinical pathology of critical patient with novel coronavirus pneumonia (COVID-19), Preprints 2020, 2020020407.

3. Xiong Y, Sun D, Liu Y, Fan Y, Zhao L, Li X et al (2020) Clinical and high-resolution CT features of the COVID-19 infection: comparison of the initial and follow-up changes. Investig Radiol. https://doi.org/10.1016/j.acra.2020.03.002

4. National Health Commission of the People's Republic of China. Technical guideline for the laboratory test of pneumonia caused by a novel coronavirus (trial version 7). http://www.nhc.gov.cn/yzygj/ s7653p/202003/46c9294a7dfe4cef80dc7f5912eb1989/files/ ce3e6945832a438eaae415350a8ce964.pdf, Published on March 3, 2020, Accessed date: 3 April 2020.

5. Liu F, Zhang Q, Huang C, Shi C, Wang L, Shi N et al (2020) CT quantification of pneumonia lesions in early days predicts progression to severe illness in a cohort of COVID-19 patients. Theranostics 10(12):5613-5622. https://doi.org/10.7150/thno. 45985

6. ACR recommendations for the use of chest radiography and Computed tomography (CT) for suspected COVID-19 infection. https://www.acr.Advocacy-and-Economics/ACR-PositionStatements/Recommendations-for-Chest-Radiography-and-CTfor-Suspected-COVID19-Infection.

7. Pan F, Ye T, Sun P et al (2020) Time course of lung changes on chest CT during recovery from 2019 novel coronavirus (COVID19) pneumonia. Radiology 13:200370. https://doi.org/10.1148/ radiol.2020200370.M

8. Chung M, Bernheim A, Mei X, Zhang N, Huang M, Zeng X et al (2020) CT imaging features of 2019 novel coronavirus (2019nCoV). Radiology 295:200230. https://doi.org/10.1148/radiol. 2020200230

9. LL Qi BTW, Tang W, Zhou LN, Huang Y, Zhao SJ et al (2020) Long-term follow-up of persistent pulmonary pure ground-glass nodules with deep learning-assisted nodule segmentation. Eur Radiol 30(2):744-755. https://doi.org/10.1007/s00330-01906344-Z

10. Chassagnon G, Vakalopoulou M, Paragios N, Revel MP (2020) Artificial intelligence applications for thoracic imaging. Eur J Radiol 123:108774. https://doi.org/10.1016/j.ejrad.2019.108774

11. Li M, Lei P, Zeng B et al (2020) Coronavirus disease (COVID-19): spectrum of CT findings and temporal progression of the disease. Acad Radiol. https://doi.org/10.1016/j.acra.2020.03.003

12. National Health Commission of the People's Republic of China. Diagnosis and treatment protocols pneumonia caused by a novel coronavirus (trial version 3). http://www.nhc.gov.cn/yzygj/s7653p/ $202001 /$ f 492 c9 153 ea 9437 bb 587 ce 2 ffcbee 1 fa/files/ 39e7578d8596be81117736dd789d8f.pdf, Published on January 22, 2020. Accessed date: 3 April 2020. 
13. Li Z, Zhang S, Zhang J, Huang K (2019) MVP-Net: Multi-view FPN with position-aware attention for deep universal lesion detection. In: Shen D, Liu T, Peters TM et al (eds) Medical Image Computing and Computer Assisted Intervention - MICCAI 2019. Vol 11769. Springer International Publishing, Cham, pp 13-21

14. Ronneberger O, Fischer P, Brox T (2015) U-net: Convolutional networks for biomedical image segmentation[C]//International Conference on Medical image computing and computer-assisted intervention. Springer, Cham, pp 234-241

15. Li F, Li W, Farzan M, Harrison S (2005) Structure of SARS coronavirus spike receptor-binding domain complexed with receptor. Science 309(5742):1864-1868. https://doi.org/10.1126/science. 1116480

16. Zhao Y, Zhao Z, Wang Y, Zhou Y, Ma Y, Zuo W (2020) Singlecell RNA expression profiling of ACE2, the putative receptor of Wuhan 2019-nCov. BioRxiv 202. https://doi.org/10.1101/2020.01. 26.919985

17. Wong KT, Antonio GE, Hui DSC, Lee N, Ahuja AT (2003) Severe acute respiratory syndrome: radiographic appearances and pattern of progression in 138 patients. Radiology 228(2):401-406. https:// doi.org/10.1148/radiol.2282030593

18. Shen C, Yu N, Cai S, Zhou J, Sheng J, Liu K et al (2020) Quantitative computed tomography analysis for stratifying the severity of coronavirus disease 2019. J Pharm Anal 10(2):123-129. https://doi.org/10.1016/j.jpha.2020.03.004

19. Cheng Z, Qin L, Cao Q, Dai J, Pan A, Yang W et al (2020) Quantitative computed tomography of the coronavirus disease 2019 (COVID-19) Pneumonia. Radiol Infect Dis 7(2):55-61. https://doi.org/10.1016/j.jrid.2020.04.004

20. Wang Y, Chen Y, Wei Y, Li M, Zhang Y, Zhang N et al (2020) Quantitative analysis of chest $\mathrm{CT}$ imaging findings with the risk of
ARDS in COVID-19 patients: a preliminary study. Ann Trans1 Med 8(9):594. https://doi.org/10.21037/atm-20-3554

21. Salehi S, Abedi A, Balakrishnan S, Gholamrezanezhad A (2020) Coronavirus disease 2019 (COVID-19): a systematic review of imaging findings in 919 Patients. AJR Am J Roentgenol 215:1-7. https://doi.org/10.2214/AJR.20.23034

22. Xu X, Yu C, Qu J, Zhang L, Jiang S, Huang D (2020) Imaging and clinical features of patients with 2019 novel coronavirus SARSCoV-2. Eur J Nucl Med Mol Imaging 47:1-6. https://doi.org/10. 1007/s00259-020-04735-9

23. Lei J, Li J, Li X, Qi X (2020 Apr) CT imaging of the 2019 novel coronavirus (2019-nCoV) pneumonia. Radiology. 295(1):18. https://doi.org/10.1148/radiol.2020200236

24. Duan YN, Qin J (2020) Pre- and posttreatment chest CT findings: 2019 novel coronavirus (2019-nCoV) pneumonia. Radiology 295(1):21. https://doi.org/10.1148/radiol.2020200323

25. Huang G, Gong T, Wang G, Wang J, Guo X, Cai E et al (2020) Timely diagnosis and treatment shortens the time to resolution of coronavirus disease (COVID-19) pneumonia and lowers the highest and last CT scores from sequential chest CT. AJR Am J Roentgenol 215(2):367-373. https://doi.org/10.2214/AJR

26. Sun D, Li X, Guo D, Wu L, Chen T, Fang Z et al (2020) CT quantitative analysis and its relationship with clinical features for assessing the severity of patients with COVID-19. Korean J Radiol 21. https://doi.org/10.3348/kjr.2020.0293

Publisher's note Springer Nature remains neutral with regard to jurisdictional claims in published maps and institutional affiliations. 